\title{
EMPUXO SOBRE UM CORPO IMERSO: UMA INVESTIGAÇÃO EM CONTEXTO DE APRENDIZAGEM ATIVA CONSIDERANDO 0 PARADOXO HIDROSTÁTICO DE GALILEU
}

\author{
BUOYANCY ON A BODY IMMERSED: AN INVESTIGATION IN THE CONTEXT \\ OF ACTIVE LEARNING CONSIDERING THE GALILEU'S PARADOX
}

DOI: http://dx.doi.org/10.23926/RPD.2526-2149.2019.v4.n1.p185-195.id366

\section{Erick dos Santos Silva \\ Doutorando em Ensino de \\ Ciências (USP) \\ Professor do IFMT - Campus \\ Juína \\ ssf.erick@gmail.com}

Alexandre Alberto
Visentin Ramos de
Araujo
Mestre em Ensino de
Ciências (IFRJ)
Professor do Colégio Militar
de Juiz de Fora
$\underline{\text { alexandrevisentin@gmail.co }}$
$\underline{\mathrm{m}}$

Resumo: Este trabalho apresenta um conjunto de atividades, realizadas em um curso superior de biologia da rede federal de ensino, com objetivo de investigar o aprendizado do empuxo em contextos de aprendizagem ativa. Os discentes foram apresentados a uma configuração experimental na qual o conceito de empuxo, conforme enunciado em livros didáticos de ensino médio e superior, falha. Tal configuração é conhecida como paradoxo de Galileu. Apesar da crítica apontada no século XVII, imprecisões de linguagem continuam ocorrendo, até mesmo entre estudantes de graduação, conforme mostramos no presente texto. Tais imprecisões parecem ser reforçadas por obras consagradas da literatura especializada em ensino de física.

Palavras-chave: Empuxo; Paradoxo hidrostático; Aprendizagem ativa.

\begin{abstract}
This work presents a set of activities, carried out in a biology course of the federal education network, with the objective of investigating the learning of the buoyancy in active learning contexts. The students were presented with an experimental configuration in which the concept of buoyancy, as stated in high school textbooks and higher, fails. Such a configuration is known as Galileo's paradox. Despite the criticism pointed out in the seventeenth century, inaccuracies of language continue to occur, even among undergraduate students, as we show in this text. Such inaccuracies seem to be reinforced by consecrated works of literature specialized in physics teaching.
\end{abstract}

Keywords: Buoyancy; Hydrostatic paradox; Active learning. 


\section{INTRODUÇÃO}

A ideia de que um corpo imerso em um fluido fica sujeito a uma força vertical para cima é conhecida há muito tempo. Arquimedes (287 a.C - 212 a.C.) teria descoberto esse fato, enunciado comumente nos livros de física em forma de princípio: "todo corpo mergulhado em um líquido sofre um empuxo de baixo para cima igual ao peso do fluido por ele deslocado". Conforme exposto em Silveira e Medeiros (2009), os arranjos experimentais relativos ao conceito de empuxo, apresentados em livros didáticos de ensino médio e superior, parecem convincentes e são baseados em trabalhos de Gravezande (1688-1742) e Musschenbroek (16921761), datados do século XVIII (WILSON, 1995; BARTLETT, 1997; SILVEIRA; MEDEIROS, 2009).

Contudo, uma imprecisão teria sido apontada por Galileu Galilei (1564-1642), ainda no século XVII. Essa crítica origina o chamado paradoxo hidrostático de Galileu: um corpo é capaz de flutuar em um líquido mesmo quando o peso de líquido disponível é menor do que o peso do corpo. O paradoxo é estabelecido observando que o corpo pode flutuar mesmo quando o volume deste fluido é menor do que o corpo "precisaria deslocar" para atender ao enunciado atribuído a Arquimedes (WILSON, 1995; BARTLETT, 1997; SILVEIRA; MEDEIROS, 2009).

A fim de verificar essa contradição, exploramos o enunciado comumente encontrado para o princípio de Arquimedes a partir de uma abordagem experimental, por meio de atividades realizadas com alunos do primeiro semestre de um curso de licenciatura em ciências biológicas da rede federal de ensino, localizado em Mato Grosso. Os discentes cursavam a disciplina "Física Geral e Experimental (FGE)", cuja ementa propõe a discussão do empuxo. O objetivo das atividades desenvolvidas foi oferecer uma análise fenomenológica mais rigorosa para os estudantes de graduação envolvidos. Desse modo, era esperado que as aulas de física, durante as quais as atividades foram realizadas, fossem capazes de minimizar as imprecisões de linguagem apresentadas por alguns alunos, decorrentes de concepções alternativas reforçadas por textos presentes em livros didáticos (SILVEIRA; MEDEIROS, 2009; CHIQUETTO; KRAPAS, 2012; RAMALHO; FERRARO; SOARES, 2007; HALLIDAY; RESNICK; WALKER, 2008). Esse tipo de abordagem pode contribuir para a formação acadêmica do estudante que ingressa no curso superior do campus, ao revelar um interessante caso de análise reducionista propagada em textos didáticos sobre empuxo (WILSON, 1995; BARTLETT, 1997; SILVEIRA; MEDEIROS, 2009). 


\section{APRENDIZAGEM ATIVA E LIVROS DIDÁTICOS NO ENSINO SUPERIOR}

\subsection{Alusão ATUAL À APRENDiZAGEM ATIVA}

A partir da década de 80 , estudos sistemáticos sinalizaram para um baixo aproveitamento dos estudantes nas disciplinas de física, quando submetidos apenas às aulas expositivas tradicionais (HALLOUN; HESTENES, 1985; HAKE, 1998; HENRIQUES; PRADO; VIEIRA, 2014). Essas aulas apresentam, praticamente, uma via de mão única, do professor para o aluno, onde o docente é responsável pela síntese dos conteúdos que devem ser apresentados e aprendidos. As aulas são uma espécie de "palestra", onde bastaria ouvir bons palestrantes para o processo de aprendizagem transcorrer de forma eficaz.

Dois apontamentos ficaram claros nesses estudos. O primeiro está associado ao fato de que os estudantes aprendem muito precariamente os conceitos físicos, independente das habilidades matemáticas que possuem. O segundo está relacionado com a pouca efetividade das aulas expositivas clássicas na reversão desse cenário de baixa aprendizagem conceitual (HENRIQUES; PRADO; VIEIRA, 2014).

Com possíveis inspirações em trabalhos datados do final do século XIX e início do século XX, algumas iniciativas recentes têm sido apresentadas para contornar esses problemas relatados sistemática pela literatura (GAUTHIER; TARDIF, 2014). Em Havard, por exemplo, o pesquisador e professor de física Eric Mazur, propôs o método Peer Instruction ou "instrução pelos pares" em tradução usual. A ideia central deste processo é a aprendizagem por meio da interação entre os estudantes, considerando contextos de aprendizagem ativa (ARAUJO, et al., 2017; HENRIQUES; PRADO; VIEIRA, 2014; MAZUR, 1997). Nesses contextos, os estudantes participariam de forma mais engajada no processo ensino-aprendizagem, não sendo apenas espectadores das aulas expositivas ministradas pelos docentes.

Outras metodologias surgiram, em perspectiva semelhante, como o SCALE-UP (Student-centered active learning environment with upside-down pedagogies), de Robert Beichner, e, sua variante, denominada TEAL (technology-enhanced active learning). Esses exemplos representam implementações ativas bem sucedidas em instituições renomadas como MIT e Yale (ARAUJO, et al., 2017; MÜLLER et al., 2017).

No Brasil, as metodologias ativas têm sido pesquisadas, com vigor semelhante. Por exemplo, em 2004, Barros e colaboradores (2004) reformularam o curso de Física I da Universidade Federal de Juiz de Fora (UFJF). O objetivo era promover um ambiente mais ativo, 
avaliando o processo de ensino-aprendizagem estabelecido. A postura mais ativa modelada para os estudantes foi denominada, no trabalho, de engajamento interativo (BARROS et al., 2004).

Ainda no ensino brasileiro, Oliveira et al. (2015) relatam uma experiência combinando o "Ensino sob medida" com o "Peer Instrcuction" para a abordagem de tópicos de eletromagnetismo no nível médio (OLIVEIRA et al., 2015). A atividade foi realizada com uma turma de ensino médio, sendo o processo de ensino-aprendizagem avaliado como positivo pelos autores.

Cabe destacar, que no método de Mazur, o Peer Instruction, as aulas são conduzidas por meio da discussão de questões conceituais, onde os alunos escolhem de forma individual as respostas que julgam corretas. Após essa primeira escolha, os estudantes discutem entre si as diferentes respostas, apresentando os argumentos que conduziram à defesa da mesma. Tal processo permite que cada um altere ou continue com suas respostas iniciais, em relação à indagação conceitual apresentada pelo professor. Na sequência, os discentes apresentam novamente suas respostas, que podem ter sido alteradas ou não. Desse modo, o professor é capaz de coletar as respostas dos estudantes antes e depois das discussões de sala, comparando o número de acertos obtidos em cada questão. Vale destacar que esse processo de coleta pode ser realizado de forma automatizada e os acertos podem ser avaliados estatisticamente (ARAUJO et al., 2017).

Já na metodologia de "Ensino sob medida" (Just in Time Teaching), um conjunto de atividades iniciais é proposta aos alunos e, a partir dos resultados dessa atividade, o professor prepara uma aula "sob medida" para a classe. Isso permite focar nas dificuldades específicas apresentadas pelas diferentes turmas (OLIVEIRA et al., 2015).

Já em 2017, Araujo e colaboradores (2017) aplicaram a metodologia Peer Instruction para o estudo de circuitos elétricos, apontando para uma ponderação necessária sobre os parâmetros de ganho (ganho de Hake) utilizados nos contextos brasileiros (ARAUJO et al., 2017). O ganho de Hake permite avaliar o impacto de uma sequência didática aplicada em determinada turma, considerando os resultados dos alunos em um protocolo pré-pós testes. Os parâmetros obtidos em contextos nacionais são relativamente diferentes daqueles relatados por Mazur, por exemplo (ARAUJO et al., 2017). Conforme discutimos naquela oportunidade, as especificidades de cada contexto, como o nível educacional envolvido, por exemplo, devem ser exploradas com cuidado, a fim de se evitar comparações espúrias. Maiores detalhes sobre esse trabalho e outros envolvendo o Peer Instruction podem ser encontrados em Araujo e 
colaboradores (2017) e no artigo de revisão de Müller e colaboradores (2017) (ARAUJO et al., 2017; MÜLLER et al., 2017).

\subsection{LIVROS DIDÁTICOS NO CONTEXTO DO ENSINO SUPERIOR}

O governo federal tem realizado esforços significativos para a compra e distribuição de livros didáticos nas redes públicas de ensino básico, considerando os níveis fundamental e médio (ZAMBON; TERRAZZAN, 2017). O livro didático pode ser considerado, atualmente, um recurso instrucional relativamente presente nas instituições brasileiras, sendo, muitas vezes, o principal balizador das atividades desenvolvidas em sala de aula (GARCIA, 2012; NETO; FRACALANZA, 2003). No entanto, como fica a situação do ensino superior?

De fato, a pesquisa em ensino tem reforçado a necessidade de se ampliar os estudos sobre o livro didático, considerando sua produção, utilização e papel na relação ensinoaprendizagem atual. Alguns autores relatam mudanças nos livros didáticos ao longo do tempo (GARCIA, 2012; DOMINGUINI, 2012; NETO; FRACALANZA, 2003), que buscam atender às especificações de documentos legais. É importante ressaltar que o foco destes estudos geralmente está na educação básica (GARCIA, 2012; DOMINGUINI, 2012; NETO; FRACALANZA, 2003).

No entanto, o papel do livro didático é tema de grandes divergências entre autores da área de ensino (ZAMBON; TERRAZZAN, 2017). A complexidade de se analisar uma obra didática é clara, podendo ser sintetizada em perguntas do tipo: Em que medida os livros ainda precisam melhorar? Qual seria o real papel do livro didático no cenário educacional atual? Enfim, no âmbito do ensino superior, o que a pesquisa tem relatado? Essa última pergunta sinaliza para a necessidade de mais estudos com o referido enfoque.

De modo geral, os cursos de física básica têm sido estruturados em torno de algumas poucas obras clássicas, como o livro Fundamentos de Física, dos autores David Halliday, Robert Resnick e Jearl Walker (HALLIDAY; RESNICK; WALKER, 2008). Isso é bom? O que os especialistas dizem? Não é objetivo deste trabalho refletir profundamente sobre essas perguntas, mas é notório que os cursos de física básica apresentam, em geral, referências bibliográficas centradas nesta obra ou em outras similares. Nesse contexto, acertos e erros dessas obras, escritas décadas atrás, continuam influenciando os atuais graduandos, alguns deles, futuros professores. Não seria interessante aprofundarmos esse tipo de investigação? O que, de fato, mudou ou precisa ser mudado? Qual o motivo da aprendizagem conceitual ser tão baixa, ainda? 
A necessidade de se acompanhar as mudanças sobre as quais os livros passam, ainda que pequenas, pode ajudar na melhoria das situações de aprendizagem estabelecidas (GARCIA, 2012; NETO; FRACALANZA, 2003; ZAMBON; TERRAZZAN, 2017). A educação superior forma futuros professores que, provavelmente, atuarão na seleção de materiais didáticos em cursos presenciais e à distância. Em que medida seus repertórios permitirão, de fato, realizar escolhas? Como os cursos de formação podem contribuir nessa direção?

\section{ESTRUTURA DA ATIVIDADE: MATERIAIS E METODOLOGIA}

A discussão a respeito da força de empuxo foi realizada por meio de uma atividade orientada pelos pressupostos da aprendizagem ativa (MAZUR, 1997; HENRIQUES; PRADO; VIEIRA, 2014; ARAUJO, et al., 2017; MULLER et al., 2017). Os estudantes foram colocados frente ao paradoxo hidrostático de Galileu, discutindo-o a partir de uma abordagem experimental. A observação do fenômeno e as interações com os colegas podem, por hipótese, auxiliar no desenvolvimento das habilidades argumentativas dos alunos participantes (HENRIQUES; PRADO; VIEIRA, 2014; ARAUJO, et al., 2017; MULLER et al., 2017; BARROS et al., 2004).

Mais especificamente, a atividade experimental consistiu em discutir o empuxo, por meio de uma configuração onde um corpo de volume $\mathrm{V}_{1}$ é inserido em um recipiente com volume $\mathrm{V}_{2}$ de fluido, sendo $\mathrm{V}_{2}<\mathrm{V}_{1}$ (Figura 1). Se o corpo de volume $\mathrm{V}_{1}$ flutuar, estando quase que completamente imerso, o enunciado usual para o empuxo falha, pois o empuxo não pode ser o "peso do fluido deslocado", nesse caso (SILVEIRA; MEDEIROS, 2009).

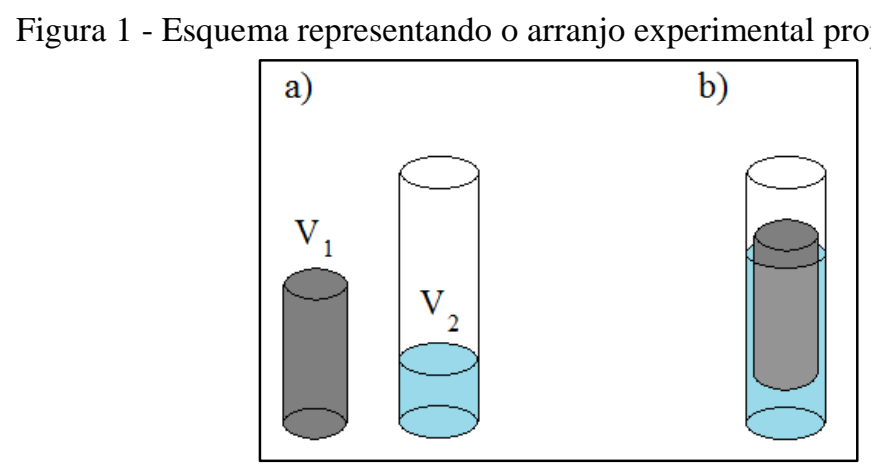

Fonte: Elaborada pelos autores.

Inicialmente, uma atividade foi proposta para os estudantes, considerando o estudo das forças sobre corpos imersos em fluidos, no caso, em água líquida. Eles deveriam, em pequenos grupos, descrever o motivo de um corpo imerso parecer mais "leve". Nesse primeiro momento, também foi descrito um arranjo experimental, nas condições do paradoxo hidrostático, onde o 
volume de fluido contido no recipiente $\left(\mathrm{V}_{2}\right)$ é menor do que o volume do corpo que será imerso $\left(\mathrm{V}_{1}\right)$ (Figura 1).

Em um segundo encontro, os grupos apresentaram os resultados da pesquisa indicada, juntamente com o esquema experimental proposto. Após a apresentação dos grupos, o conceito de empuxo foi discutido em sala de aula, com mediação do professor.

Finalmente, em um terceiro encontro, os alunos apresentaram novamente seus experimentos e descrições sobre o empuxo, inclusive em relação à configuração proposta. $\mathrm{O}$ Quadro 1 resume os três encontros estruturados nessa sequência didática.

Estabelecemos três critérios para avaliar a participação dos grupos na atividade estruturada: construção do arranjo experimental proposto $(\mathrm{C} 1)$; definição do empuxo para situações onde $\mathrm{V}_{1}<\mathrm{V}_{2}(\mathrm{C} 2)$ e definição do empuxo no limite do paradoxo hidrostático (C3). Os critérios foram avaliados como: satisfatório (S), parcialmente satisfatório (PS) e insatisfatório (I).

Quadro 1 - Atividades componentes da sequência didática. Cada encontro é composto por dois tempos de aula seguidos, totalizando 100 minutos

\begin{tabular}{|c|c|}
\hline Encontro & Atividade programada \\
\hline 1 & $\begin{array}{c}\text { Discussão inicial sobre o equilíbrio de corpos imersos em líquidos, proposição } \\
\text { da pesquisa (P1) e proposição do arranjo experimental (A1) para o estudo do } \\
\text { paradoxo hidrostático. }\end{array}$ \\
\hline 2 & $\begin{array}{r}\text { Apresentação do arranjo experimental (A1) e da pesquisa (P1) pelos 4 grupos. } \\
\text { Discussão conjunta sobre o empuxo. }\end{array}$ \\
\hline 3 & $\begin{array}{c}\text { Apresentação do arranjo experimental (A2) e da pesquisa (P2), após a } \\
\text { discussão realizada no encontro anterior e de uma nova pesquisa livre. }\end{array}$ \\
\hline
\end{tabular}

Fonte: Elaborado pelos autores.

\section{ReSultados E DiscuSSõeS}

Na etapa 1, referente à pesquisa sobre a razão de corpos imersos se apresentarem "mais leves", os grupos trouxeram explicações semelhantes, mencionando o empuxo. A ideia central pode ser sintetizada pelo seguinte texto: “O corpo imerso em água fica mais leve (peso aparente) pelo fato de uma força vertical e dirigida para cima, denominada empuxo, atuar sobre ele. $\mathrm{O}$ módulo do empuxo é igual ao peso do volume de fluido deslocado".

Em relação ao arranjo experimental, apenas um grupo (25\%) não conseguiu apresentálo satisfatoriamente. A montagem apresentada na Figura 2d não reproduziu a configuração do paradoxo hidrostático, sendo considerada insatisfatória. A montagem trazida por cada grupo pode ser conferida na Figura 2. 
Figura 2 - Esquema representando o arranjo experimental proposto aos alunos

a)

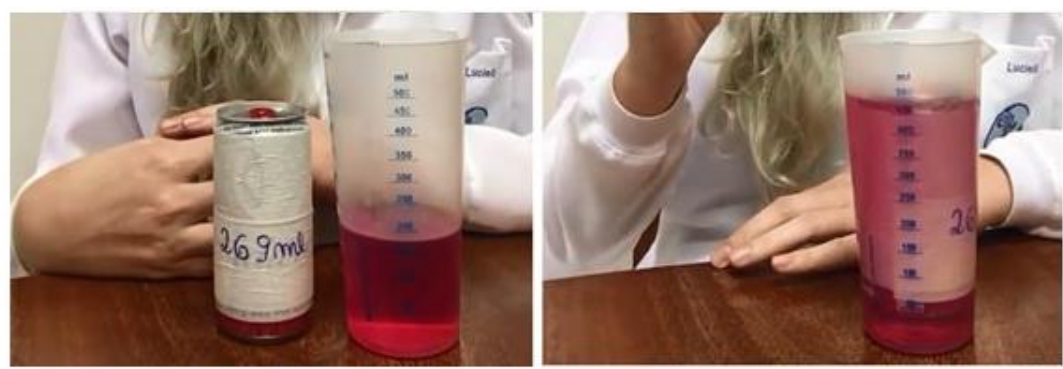

b)

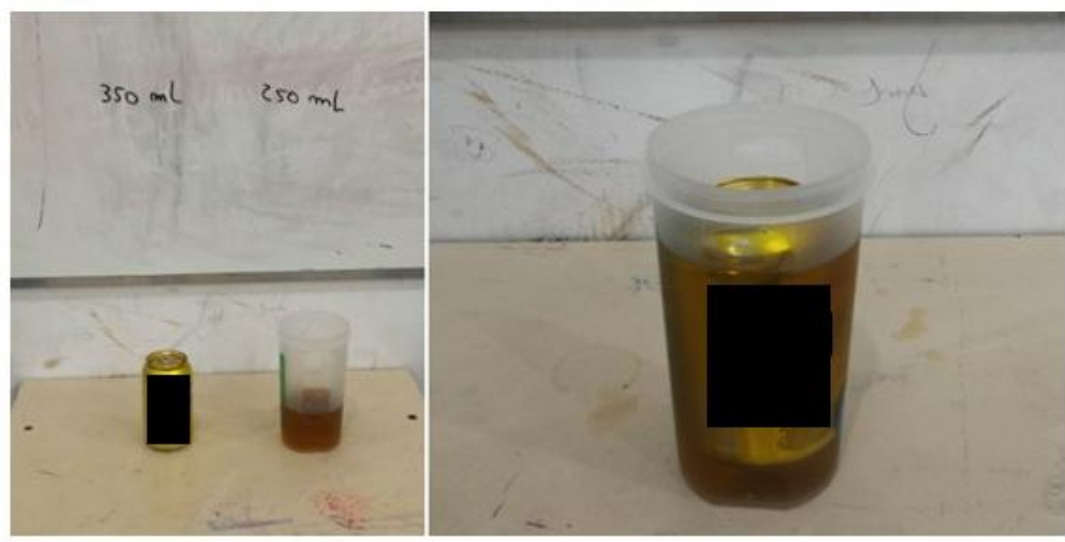

c)

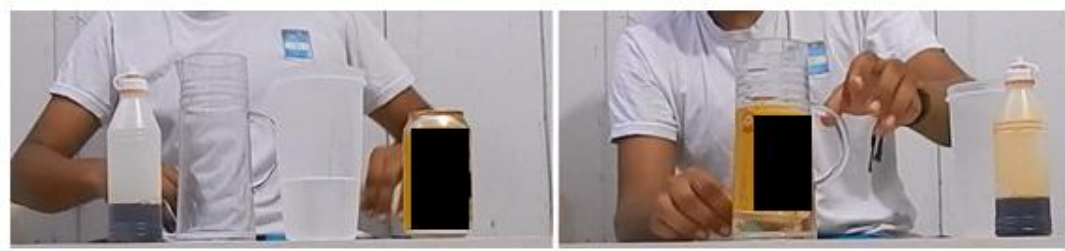

d)

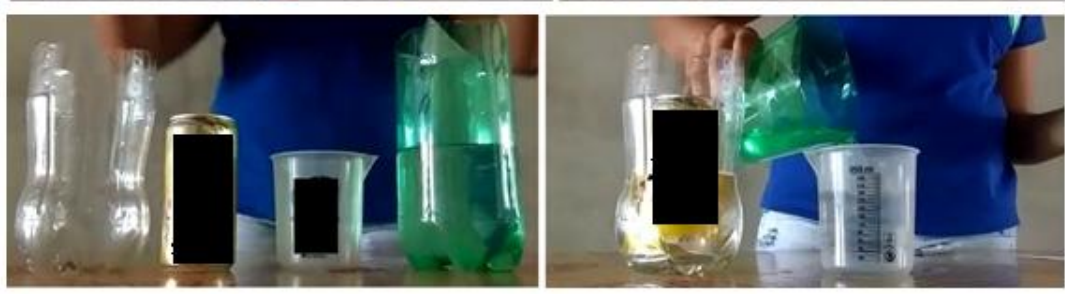

Fonte: Elaborada pelos autores.

Após a realização dos experimentos em sala, os alunos foram convidados a enunciar o empuxo, atentando para o fato de que o objeto imerso apresentava volume maior do que o volume de líquido no recipiente. Nenhum grupo foi capaz, ao longo da aula (ou seja, no segundo encontro) de apresentar um enunciado preciso para o experimento nesse limite. Todos mencionaram que o empuxo seria igual ao peso do fluido deslocado, caracterizando todas as respostas como insatisfatórias.

Os grupos tiveram a oportunidade de melhorar seus experimentos em horário extraclasse, trazendo-os para discussão em um terceiro encontro. Dessa forma, os discentes contaram com mais tempo para refletir sobre a atividade realizada, aprimorando o arranjo 
experimental e as explicações sobre o empuxo, considerando, inclusive, a configuração experimental proposta referente ao paradoxo hidrostático.

Finalmente, no último encontro, constatamos que um grupo enunciou corretamente o empuxo no limite experimental indicado (25\%), citando a referência Silveira e Medeiros (2009). O grupo mencionado expressou precisamente a ideia de que: "Todo corpo mergulhado em um líquido sofre um empuxo de baixo para cima igual ao peso do fluido contido em um volume idêntico ao volume submerso do corpo no fluido.” (SILVEIRA; MEDEIROS, 2009). Os demais grupos (75\%) continuaram mencionando o princípio comumente encontrado nos livros didáticos de ensino médio e superior (RAMALHO; FERRARO; SOARES, 2007; HALLIDAY; RESNICK; WALKER, 2008). O quadro abaixo (Quadro 2) resume os resultados descritos nessa seção, considerando o desempenho dos grupos segundo os critérios $\mathrm{C} 1, \mathrm{C} 2 \mathrm{e}$ C3, definidos para a análise da sequência didática.

Quadro 2 - Desempenho dos grupos em relação aos critérios avaliativos estabelecidos

\begin{tabular}{|c|c|c|c|}
\hline Critério avaliativo & S & PS & I \\
\hline C1 & $3 / 4$ & $0 / 4$ & $1 / 4$ \\
\hline C2 & $4 / 4$ & $0 / 4$ & $0 / 4$ \\
\hline C3 & $1 / 4$ & $0 / 4$ & $3 / 4$ \\
\hline
\end{tabular}

Fonte: Elaborada pelos autores.

De fato, percebe-se que os alunos discutem as atividades experimentais realizadas buscando, sempre que possível, um apoio literário para as suas conclusões. Contudo, em um ambiente investigativo mais aberto, não necessariamente contemplado nos livros didáticos, percebemos uma certa dificuldade dos discentes em analisar as situações experimentais estruturadas. O próprio grupo que atende ao critério $\mathrm{C} 3$ revela explicitamente que a referência Silveira e Medeiros (2009) foi fundamental para o embasamento de suas conclusões (SILVEIRA; MEDEIROS, 2009).

Vale destacar que a literatura internacional tem discutido o desafio de se promover ambientes de investigação mais autênticos, conectados com demandas contemporâneas de formação discente (MANZ; SUÁREZ, 2018[1]; BJØRKVOLD; BLIKSTAD-BALAS, 2018[1]). Nesse contexto, podemos considerar importante a busca dos alunos por suportes literários para o embasamento de suas conclusões experimentais. No entanto, isso nos remete a dois problemas imediatos: (1) a busca de respaldo literário não pode funcionar como um fator alienante, onde o aluno apenas procura uma interpretação experimental ou resposta de outrem e (2) os materiais didáticos devem apresentar rigor científico suficiente para minimizar 
possíveis imprecisões de linguagem, já que possuem forte influência nas análises promovidas pelos alunos.

\section{CONSIDERAÇÕES FINAIS}

A atividade revela que imprecisões na linguagem científica podem persistir em estudantes no nível superior. No caso, mesmo realizando uma atividade que revela uma situação onde o enunciado comumente apresentado em livros didáticos para o empuxo falha, os alunos acabam seguindo a literatura tradicional, reproduzindo o princípio que faz alusão ao "peso do fluido deslocado". Dessa forma, os estudantes seguem as principais obras de ensino médio e superior, ignorando, assim como elas, uma imprecisão apontada no século XVII por Galileu Galilei.

\section{REFERÊNCIAS}

ARAUJO, A. V. R. de; SILVA, E. S.; JESUS, V. L. B. de. OLIVEIRA, A. L. de. Uma associação do método Peer Instruction com circuitos elétricos em contextos de aprendizagem ativa. Revista Brasileira de Ensino de Física, São Paulo, v.39, n.2, e2401, 2017.

ARAUJO, A. A. V. R. ; SILVA, E. S. ; V. L. B. de Jesus ; OLIVEIRA, A. L. DE . Uma estratégia de aprendizagem ativa com atividades teórico-experimentais sobre circuitos elétricos. In: Alexandre Lopes de Oliveira e Valeria da Silva Vieira. (Org.). Nossos talentos: 10 anos do PROPEC / IFRJ. 1ed.Nilópolis: Entorno, 2017, v. 1, p. 277-292.

BARROS, J. A.; REMOLD, J.; GLAUCO, S. F. da S.; TAGLIATI, J. R. Engajamento interativo no curso de Física I da UFJF. Revista Brasileira de Ensino de Física, São Paulo, v. 26, n. 1, p. 63-69, 2004.

BARTLETT, A. The Hydrostatic Paradox revisited. The Physics Teacher, v. 35, n. 5, p. 288289, 1997.

BJØRKVOLD, T.; BLIKSTAD-BALAS, M. Students as researchers: What and why seventhgrade students choose to write when investigating their own research question. Science Education, v.102, n.2, 304-341, 2018.

CHIQUETTO, M. J.; KRAPAS, S. Livros didáticos baseados em apostilas: como surgiram e por que foram amplamente adotados. Revista Brasileira de Pesquisa em Educação em Ciências, v. 12, n. 3, 2012.

DOMINGUINI, L. Física moderna no Ensino Médio: com a palavra os autores dos livros didáticos do PNLEM. Revista Brasileira de Ensino de Física, v.34, n.2, 2502, 2012.

GARCIA, N.M.D. Livro didático de Física e de Ciências: contribuições das pesquisas para a transformação do ensino. Educar em Revista, n. 44, p. 145-163, abr/jun, 2012. 
GAUTHIER, C.; TARDIF, M. A pedagogia: Teorias e práticas da Antiguidade aos nossos dias. 3a ed. Rio de Janeiro: Ed. Vozes, 2014.

HAKE, R.R. Interactive-engagement versus traditional methods: A six-thousand student survey of mechanics test data for introductory physics courses. American Journal of Physics, v.66, 64-74, 1998.

HALLIDAY, D.; RESNICK, R.; WALKER, J. Fundamentals of Physics. 8 ed. John Wiley \& Sons, 2008. v. 2.

HALLOUN, I. A.; HESTENES, D. Common sense concepts about motion. American Journal of Physics, v.53, 1056-1065, 1985.

HENRIQUES, V. B.; PRADO, C. P. C.; VIEIRA, A. P. Editorial convidado: Aprendizagem ativa. Revista Brasileira de Ensino de Física, São Paulo, v.36, n.4, 4001, 2014.

MAZUR, E. Peer Instruction: A User's Manual. Prentice Hall, Upper Saddle River, 1997, v. 1 .

MANZ, Eve; SUÁREZ, Enrique. Supporting teachers to negotiate uncertainty for science, students, and teaching. Science Education. v.102, n.4, 711, 2018.

MULLER, M. G.; ARAUJO, I. S.; VEIT, E. A.; SCHELL, J. Uma revisão da literatura acerca da implementação da metodologia interativa de ensino Peer Instruction (1991 a 2015).

Revista Brasileira de Ensino de Física, v. 39, n. 3, 2017.

NETO, J.M.; FRACALANZA, H. O livro didático de ciências: problemas e soluções. Ciência \& Educação, v. 9, n. 2, p. 147-157, 2003.

OLIVEIRA, V., VEIT, E. A.; ARAUJO, I. S. Relato de experiência com os métodos Ensino sob Medida (Just-in-Time Teaching) e Instrução pelos Colegas (Peer Instruction) para o Ensino de Tópicos de Eletromagnetismo no nível médio. Caderno Brasileiro de Ensino de Física, v. 32, n.1, p. 180-206, 2015.

RAMALHO, F.; FERRARO, N. G.; SOARES, P. A. T. Os fundamentos da Física. 9 ed. São Paulo: Moderna, 2007. v 1.

SILVEIRA, F. L. da; MEDEIROS, A. O paradoxo hidrostático de Galileu e a lei de Arquimedes. Caderno Brasileiro de Ensino de Física, v. 26, n. 2, p. 273-294, 2009.

WILSON, A. The Hydrostatic Paradox. The Physics Teacher. v. 33, n. 8, p. 538-539, 1995.

ZAMBON, L.B.; TERRAZZAN, E.A. Livros didáticos de física e sua (sub)utilização no ensino médio. Revista Ensaio - Pesquisa em Educação em Ciências, v.19, e2668, 2017.

Recebido em:26 de fevereiro de 2019.

Aprovado em: 15 de maio de 2019. 